National Aeronautics and Space Administration Grant NGR-39-007-025

\title{
EXTERNAL ELLIPTICAL CRACK IN ELASTIC SOLID
}

$I$
$N$
$S$
$T$
$I$
$T$
$U$
$T$
$E$
$O$
$F$

by

M.K. Kassir

G.C. Sih

\section{Technical Report No. 2}

7

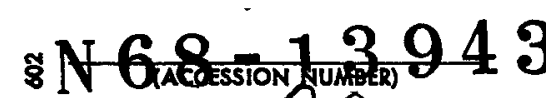

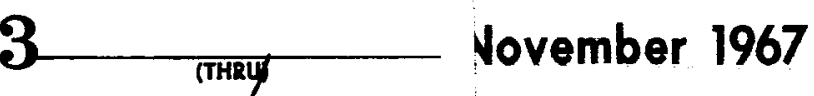

$\mathrm{R}$ 


$$
\begin{aligned}
& \text { National Aeronautics } \\
& \text { and } \\
& \text { Space Administration }
\end{aligned}
$$$$
\text { Grant NGR-39-007-025 }
$$

Technical Report No. 2

\title{
EXTERNAL ELLIPTICAL CRACK IN ELASTIC SOLID$$
\text { by }
$$

M. K. Kassir and G. C. Sin

\author{
Department of Applied Mechanics \\ Lehigh University \\ Bethlehem, Pennsylvania
}

October, -1967 


\section{EXTERNAL ELLIPTICAL CRACK IN ELASTIC SOLID ${ }^{1}$}

by

M. K. Kassir and G. C. $\sin ^{3}$

\section{ABSTRACT}

Potential functions are developed for both symmetric and antisymmetric three-dimensional problems of an infinite elastic solid containing a flat crack covering the outside of an ellipse. The knowledge of these functions permits an examination of the stress and displacement fields everywhere in the cracked solid as well as in a toroidal region around the crack border. Stress-intensity factors $k_{j}(j=1,2,3)$ corresponding to the three basic modes of fracture are obtained. The results of this paper, coupled with those found previously by the authors for the problem of internal elliptical crack, are essential in making approximate estimates of $k_{j}$ - values for solids with arbitrarily-shaped planar cracks.

1 The work reported herein was supported by the National Aeronautics and Space Administration under Grant NGR-39-007-025 with Lehigh University.

${ }^{2}$ Lecturer, Department of Civil Engineering, The City College, New York, New York.

${ }^{3}$ Professor of Mechanics, Lehigh University, Bethlehem, Pennsylvania. 
In the examination of fracture failures of solids weakened by planar cracks, it is unlikely that the configuration of the initial starting crack would be an ellipse or any other idealized shape conforming to the existing curvilinear coordinate systems. Since the mathematical problem of cracks in threedimensions involving complicated crack geometry prohibits any analytical solution, it is useful to solve some relatively simple, but basic, component crack problems whose solutions may provide rough estimates of the fracture behavior of irregularly-shaped cracks. The component problems may consist of an internal and external elliptical crack embedded in the solid. By varying the ellipticity of the internal and external crack, it is possible to approximate the periphery ${ }^{4}$ of an arbitrarilyshaped crack by joining a sequence of segments of the elliptical boundary.

Green and Sneddon $[1]^{5}$ have discussed the symmetric problem of the internal elliptical crack while kassir and Sih [2] have solved the antisymmetric problem dealing with the same geometry. In this paper, exact solutions to the problem of two half-spaces connected through an elliptically-shaped region are given. The two half-spaces are either pulled apart symmetrically or sheared

\footnotetext{
4 It is assumed that the periphery of the crack is sufficiently smooth and contains no sharp edges.

${ }^{5}$ Numbers in brackets designate references at end of paper.
} 
apart antisymmetrically with respect to the crack plane. With the help of the limiting forms of ellipsoidal to polar coordinates defined locally from the crack border as established by Kassir and Sih [2], stress-intensity factors are calculated and the results are presented graphically.

\section{STATEMENT OF THE PROBLEM}

In the formulation of boundary problems of elliptic crack, it is expedient to use ellipsoidal coordinates $(\xi, n, \zeta)$ which are defined as the roots of the equation in $s$ as

$$
\frac{x^{2}}{a^{2}+s}+\frac{y^{2}}{b^{2}+s}+\frac{z^{2}}{s}=1
$$

The restrictions placed on $\xi, n, \zeta$ are

$$
-a^{2} \leq \zeta \leq-b^{2} \leq n \leq 0 \leq \xi<\infty
$$

Referring to the work of Kassir and $\operatorname{Sin}$ [2-4], they have pointed out that a wide variety of three-dimensional problems involving discontinuity in the shape of an ellipse can be solved by making use of potentials (satisfying the Laplace equation in the variables $x, y, z$ ) in the form

$$
I(x, y, z)=\int_{\xi}^{\infty} \lambda[\omega(s)] \frac{d s}{\sqrt{Q(s)}}
$$

where

$$
\omega(s)=1-\frac{x^{2}}{a^{2}+s}-\frac{y^{2}}{b^{2}+s}-\frac{z^{2}}{s}
$$

and $Q(s)=s\left(s+a^{2}\right)\left(s+b^{2}\right)$. Without exception, harmonic functions 
of the type shown in equation (1) will also be employed in the present treatment. In elastostatics, these functions are associated with the components $u_{x}, u_{y}, u_{z}$ of the displacement vector $u_{\sim}$ such that $u_{\sim}$ satisfies the Navier equation

$$
\nabla^{2} u+\frac{1}{1-2 v} \nabla \nabla \cdot u=0
$$

Here, $v$ is Poisson's ratio and the body forces are assumed to be absent.

By an "external elliptical crack", it is meant the union of two half-spaces across a plane in the shape of an ellipse. Let this plane region $\Sigma\left(x^{2} / a^{2}+y^{2} / b^{2}=1, z=0\right)$ be located in the $x y-p l a n e$ so that the $z$-axis is directed normally to $\Sigma$ and the origin of the cartesian coordinate system coincides with the center of the ellipse. In terms of ellipsoidal coordinates, the point $(x, y, o)$ inside and outside the region $\Sigma$ can be identified by $\xi=0$ and $n=0$, respectively. This problem will be split into two parts; one symmetric and the other antisymmetric with respect to the plane $z=0$. In each case, the problem reduces to one of selecting the appropriate harmonic potentials that satisfy the prescribed boundary conditions.

\section{SYMMETRIC PROBLEM}

When the stress distribution is symmetric with respect to the plane $z=0$, the equilibrium of the cracked solid can be considered under the following conditions 


$$
\begin{aligned}
& u_{z}=\sigma_{z x}=\sigma_{z y}=0, \xi=0 \\
& \sigma_{x z}=\sigma_{y y}=\sigma_{z z}=0, n=0
\end{aligned}
$$

The resultant normal force ${ }^{6}$ acting on the elliptical region $\Sigma$ is designated by

$$
P=\iint_{\Sigma}\left(\sigma_{z z}\right)_{\xi=0} d x d y
$$

For this problem, Boussinesq's solution of the elastic equations of equilibrium, equation (2), can be employed. That is

$$
\begin{aligned}
& u_{x}=(1-2 v) \frac{\partial f}{\partial x}+z \frac{\partial^{2} f}{\partial x \partial z}, \\
& u_{y}=(1-2 v) \frac{\partial f}{\partial y}+z \frac{\partial^{2} f}{\partial y \partial z}, \\
& u_{z}=-2(1-v) \frac{\partial f}{\partial z}+z \frac{\partial^{2} f}{\partial z^{2}},
\end{aligned}
$$

in which the harmonic potential $f(x, y, z)$ satisfies the Laplace equation

$$
\nabla^{2} f(x, y, z)=0
$$

The relevant stress components corresponding to the displacements in equation (5) are

${ }^{6}$ The specification of uniform stress at infinity should be avoided since it leads to unbounded tensile force across the region $\Sigma$. 


$$
\frac{\sigma x z}{2 \mu}=z \frac{\partial^{3} f}{\partial x \partial z^{2}}, \frac{\sigma y z}{2 \mu}=z \frac{\partial^{3} f}{\partial y \partial z^{2}}, \frac{\sigma z z}{2 \mu}=-\frac{\partial^{2} f}{\partial z^{2}}+z \frac{\partial^{3} f}{\partial z^{3}}
$$

with $\mu$ being the shear modulus of the elastic solid. On the plane $z=0$, it is obvious that the shear stresses $\sigma_{x z}$ and $\sigma_{y z}$ vanish while the normal stress $\sigma_{z z}$ becomes

$$
\sigma_{z z}=-2 \mu \frac{\partial^{2} f}{\partial z^{2}}, z=0
$$

As a special case of equation (1) with $\lambda[\omega(s)]=A$ (const.), let $\partial f / \partial z$ be equal to

$$
\frac{\partial f}{\partial z}=A \int_{\xi}^{\infty} \frac{d s}{\sqrt{0(s)}}=\frac{2 A}{a} u
$$

In equation ( 8$)$, $u$ is a variable related to $\xi$ by the relation

$$
\xi=a^{2}\left(\mathrm{sn}^{-2} u-1\right)
$$

and hence it takes the value of $K(k)$ at $\xi=0$, where $K(k)$ is the complete elliptic integral of the first kind associated with the argument $k^{2}=1-(b / a)^{2}$. In addition, since

$$
\frac{\partial^{2} f}{\partial z^{2}}=-\frac{2 A\left[n \zeta\left(a^{2}+\xi\right)\left(b^{2}+\xi\right)\right]^{1 / 2}}{a b(\xi-n)(\xi-\zeta)}
$$

${ }_{z z}$ vanishes automatically for $n=0$. The only remaining condition, $u_{z}=0$ for $\xi=0$, in equation (3) can be satisfied by rewriting $u_{z}$ in equation (5) as

$$
u_{z}=-2(1-v) \frac{\partial f}{\partial z}+2(1-v) A \int_{0}^{\infty} \frac{d s}{\sqrt{Q(s)}}+z \frac{\partial^{2} f}{\partial z^{2}}
$$


The additional term corresponds to a rigid body displacement and will not affect the state of stress. Now, inserting equation (9) into (7) and the result subsequently into equation (4) yield

$$
P=\frac{4 \mu A}{a b} \iint_{\Sigma} \frac{d x d y}{\left(1-x^{2} / a^{2}-y^{2} / b^{2}\right)^{1 / 2}}=8 \pi \mu A
$$

and thus

$$
A=\frac{P}{8 \pi \mu}
$$

The solution is essentially complete. Once $\partial f / \partial z$ is known, the stresses and displacements throughout the elastic solid can be calculated.

\section{ANTISYMMETRIC PROBLEM}

Suppose that the stresses in the solid are distributed antisymmetrically with respect to the plane $z=0$, then

$$
\begin{aligned}
& u_{x}=u_{y}=\sigma_{z z}=0, \xi=0 \\
& \sigma_{x z}=\sigma_{y z}=\sigma_{z z}=0, n=0
\end{aligned}
$$

In this case, the resultant shear forces transmitted across $\Sigma$ in the $x$ - and $y$-directions will be denoted by

$$
Q=\iint_{\Sigma}\left(\sigma_{x z}\right)_{\xi=0} d x d y \text {, and } R=\iint_{\Sigma}\left(\sigma_{y z}\right)_{\xi=0} d x d y
$$

A solution of Navier's equations that gives zero normal 
stress $\sigma_{z z}$ everywhere in the plane $z=0$ can be written in terms of two harmonic potentials:

$$
\begin{aligned}
& u_{x}=2 \frac{\partial g}{\partial z}-2 v \int_{z}^{\infty} \frac{\partial G}{\partial x} d z-z \frac{\partial G}{\partial x} \\
& u_{y}=2 \frac{\partial h}{\partial z}-2 v \int_{z}^{\infty} \frac{\partial G}{\partial y} d z-z \frac{\partial G}{\partial y} \\
& u_{z}=(1-2 v) G-z \frac{\partial G}{\partial z}
\end{aligned}
$$

The function $G(x, y, z)$ stands for

$$
G=\frac{\partial g}{\partial x}+\frac{\partial h}{\partial y}
$$

in which $g(x, y, z)$ and $h(x, y, z)$ satisfy

$$
\nabla^{2} g(x, y, z)=0, \nabla^{2} h(x, y, z)=0
$$

From the stress-displacement relations, it is found that

$$
\frac{\sigma x z}{2 \mu}=\frac{\partial^{2} g}{\partial z^{2}}-z \frac{\partial^{2} G}{\partial x \partial z}, \frac{\sigma y z}{2 \mu}=\frac{\partial^{2} h}{\partial z^{2}}-z \frac{\partial^{2} G}{\partial y \partial z}, \frac{\sigma z z}{2 \mu}=z \frac{\partial^{2} G}{\partial z^{2}}
$$

The stress components $\sigma_{x x}, \sigma_{y y}$, and $\sigma_{x y}$ can be found in the same way.

First, let the elliptical connection $\Sigma$ be sheared along the major axis with $R=0$. In such a case, it is appropriate to set

$$
\frac{\partial g}{\partial z}=B \int_{\xi}^{\infty} \frac{d s}{\sqrt{Q(s)}}, h(x, y, z)=0
$$


from which it can be readily verified that $\sigma_{x z}$ and $\sigma_{y z}$ vanish outside the region $\Sigma$ at $z=0$. As in the symmetric problem, the displacements $u_{x}$ and $u_{y}$ in equation (13) are modified into the forms

$$
\begin{array}{rl}
u_{x} & =2 \frac{\partial g}{\partial z}-2 B \int_{0}^{\infty} \frac{d s}{\sqrt{Q(s)}}-2 v \int_{z}^{\infty} \frac{\partial^{2} g}{\partial x^{2}} d z \\
& +2 v \int_{0}^{\infty}\left(\frac{\partial^{2} g}{\partial x^{2}}\right) \\
\xi=0 & d z-z \frac{\partial^{2} g}{\partial x^{2}}, \\
u_{y} & =-2 v \int_{z}^{\infty} \frac{\partial^{2} g}{\partial x \partial y} d z+2 v \int_{0}^{\infty}\left(\frac{\partial^{2} g}{\partial x \partial y}\right)_{\xi=0} d z-z \frac{\partial^{2} g}{\partial x \partial y}
\end{array}
$$

such that $u_{x}=u_{y}=0$ for $\xi=0$, and the stresses in equation (14) remain unchanged. Having satisfied all the boundary conditions, the constant $B$ is evaluated from equation (12) with $\sigma_{y z}=0$ for $\xi=0$. The resuitt is

$$
B=-\frac{Q}{8 \pi \mu}
$$

If the action of shear is directed along the minor axis of the ellipse $x^{2} / a^{2}+y^{2} / b^{2}=1$, then $Q=0$ in equation (12), and

$$
g(x, y, z)=0, \frac{\partial h}{\partial z}=c \int_{\xi}^{\infty} \frac{d s}{\sqrt{Q(s)}}
$$

Similarly, expressions pertaining to rigid body motions may be added onto $u_{x}$ and $u_{y}$ so as to satisfy equation (11). The constant $C$ is found to be 
$C=-\frac{R}{8 \pi \mu}$

It is clear that the two foregoing results may be superposed to render the complete solution to the problem of an external elliptical crack sheared in its own plane along any given direction.

\section{CRACK-EXTENSION FORCE}

To compute the strain-energy change for a small opening of the crack boundary or the force tending to cause crack extension, it is pertinent to obtain asymptotic expansions of the appropriate stress components about the crack border. In this way, the crack stress-field parameters $k_{j}(j=1,2,3)$ can be determined and the crack-extension forces $G_{j}(j=1,2,3)$ can then be found from the relationships ${ }^{7}$

$$
G_{1}=\frac{\pi\left(1-v^{2}\right)}{E} k_{1}^{2}, G_{2}=\frac{\pi\left(1-v^{2}\right)}{E} k_{2}^{2}, G_{3}=\frac{\pi(1+v)}{E} k_{3}^{2}
$$

where $E$ is Young's modulus of elasticity.

The parameters $k_{j}$, commonly known as the stress-intensity factors, can be evaluated from the formulas

$$
k_{1}=\lim _{r \rightarrow 0} \sqrt{2 r}\left(\sigma_{z z}\right)_{\theta=0}
$$

7 Kassir and Sih [2] have shown that the combined state of plane strain and of anti-plane deformation always prevail near the border of an embedded crack under arbitrary loadings. 


$$
\begin{aligned}
& k_{2}=\lim _{r \rightarrow 0} \sqrt{2 r}\left(\sigma_{n z}\right)_{\theta=0} \\
& k_{3}=\lim _{r \rightarrow 0} \sqrt{2 r}\left(\sigma_{t z}\right)_{\theta=0}
\end{aligned}
$$

The shear stresses $\sigma_{n z}$ and $\sigma_{t z}$ act in the directions normal and tangent to the ellipse $x^{2} / a^{2}+y^{2} / b^{2}=1$ and they can be associated with the rectangular components $\sigma_{x z}$ and $\sigma_{y z}$ by means of the following transformations [2]

$$
\begin{aligned}
& \sigma_{n z}=\left(b \sigma_{x z} \cos \phi+a \sigma_{y z} \sin \phi\right)\left(a^{2} \sin ^{2} \phi+b^{2} \cos ^{2} \phi\right)^{-1 / 2} \\
& \sigma_{t z}=\left(-a \sigma_{x z} \sin \phi+b \sigma_{y z} \cos \phi\right)\left(a^{2} \sin ^{2} \phi+b^{2} \cos ^{2} \phi\right)^{-1 / 2}
\end{aligned}
$$

More precisely, the set of axes $n, t$, and $z$ are always situated along the binormal, tangent, and principal normal of the crack boundary, respectively. The angle $\phi$ is referenced from the major axis of the ellipse as illustrated in Figures 1 and 2 . In equation (21), the radial distance $r$ is measured from the crack front in the $n z-p l a n e$ and the angle $\theta$ from the elliptical region $\Sigma$.

In view of equation (21), the evaluation of $k_{j}$ requires only the $(1 / \sqrt{2 r})$ - terms of $\sigma_{n z}$, $\sigma_{t z}$, and $\sigma_{z z}$ because the nonsingular terms vanish in the limit as $r \rightarrow 0$. These singular terms can be found from a knowledge of the limiting forms of 
$(\xi, n, \zeta)$ to $(r, \theta)^{8}:$

$$
\begin{aligned}
& \xi=2 a b r\left(\sin \frac{\theta}{2}\right)^{2}\left(a^{2} \sin ^{2} \phi+b^{2} \cos ^{2} \phi\right)-1 / 2 \\
& \eta=-2 a b r\left(\cos \frac{\theta}{2}\right)^{2}\left(a^{2} \sin ^{2} \phi+b^{2} \cos ^{2} \phi\right)^{-1 / 2} \\
& \zeta=-\left(a^{2} \sin ^{2} \phi+b^{2} \cos ^{2} \phi\right)
\end{aligned}
$$

\section{(a) Opening Mode}

With the aid of equation (23), equation (9) may be inserted into equation (7) to obtain

$$
\left(\sigma_{z z}\right)_{\theta=0}=\frac{P}{2 \pi \sqrt{a b}}\left(a^{2} \sin ^{2} \phi+b^{2} \cos ^{2} \phi\right)^{-1 / 4} \frac{1}{\sqrt{2 r}}+0\left(r^{0}\right)
$$

From equation (21), the stress-intensity factor $k_{1}$ for the opening mode is found ${ }^{9}$ :

$$
k_{1}=\frac{p}{2 \pi \sqrt{a b}}\left(a^{2} \sin ^{2} \phi+b^{2} \cos ^{2} \phi\right)^{-1 / 4}
$$

For a circular connection, $a=b$, and equation (25) simplifies to

\footnotetext{
8 For details, refer to the paper by Kassir and Sih [2], equation (55). Their definition of the angle $\theta$ is equal to $\pi$ minus that given in equation (23).

${ }^{9}$ This result was simply stated by Westmann [5] without any detailed derivations.
} 


$$
k_{1}=\frac{P}{2 \pi a^{3 / 2}}
$$

The variations of $k_{1}$ in equation (25) with the angle $\phi$ for different values of $a / b$ are plotted in Figure 1 . Note that $k_{1}$ attains its maximum value at $\phi=0, \pi$, where the crack boundary intersects the major axis of the ellipse. Based on the notion of brittle fracture theory, if the tension $P$ is sufficiently large, the elliptical bond should spread inward and tend to produce a circular bond of radius b. Any further crack propagation will reduce the radius of the circular bond to the point of complete separation of the solid into two half-spaces.

This is contrary to the situation of an internal elliptical crack. It was shown by Irwin [6] that the maximum $k_{1}$ value occurs at $\phi= \pm \pi / 2$. Hence, an internal elliptical crack will propagate outward into the solid and tend to produce a penny-shaped crack of radius a, the major semiaxis of the ellipse. A graphical representation of Irwin's result is given by Kassir and Sih [2].

From the results of the elliptical crack and bond problems, estimates can be made for stress-intensity factor for quite arbitrary crack-front contours in solids under tensile loads.

\section{(b) Shearing And Tearing Modes}

The $k_{j}$-factors $(j=2,3)$ for an elliptical connection sheared along the major axis can be computed by the same pro- 
cedure. The results of combining equations (14), (15), (22), and (23) are

$$
\begin{aligned}
& \sigma_{n z}=\frac{Q}{2 \pi} \sqrt{\frac{b}{a}} \cos \phi\left(a^{2} \sin ^{2} \phi+b^{2} \cos ^{2} \phi\right)^{-3 / 4} \frac{1}{\sqrt{2 r}}+0\left(r^{0}\right) \\
& \sigma_{t z}=-\frac{Q}{2 \pi} \sqrt{\frac{a}{b}} \sin \phi\left(a^{2} \sin ^{2} \phi+b^{2} \cos ^{2} \phi\right)-3 / 4 \frac{1}{\sqrt{2 r}}+0\left(r^{0}\right)
\end{aligned}
$$

from which $k_{j}(j=2,3)$ in equation (21) can be deduced:

$$
\begin{aligned}
& k_{2}=\frac{Q}{2 \pi} \sqrt{\frac{b}{a}} \cos \phi\left(a^{2} \sin ^{2} \phi+b^{2} \cos ^{2} \phi\right)^{-3 / 4} \\
& k_{3}=-\frac{Q}{2 \pi} \sqrt{\frac{a}{b}} \sin \phi\left(a^{2} \sin ^{2} \phi+b^{2} \cos ^{2} \phi\right)^{-3 / 4}
\end{aligned}
$$

If $a=b$, equation (27) reduces to the $k_{j}$-values of a pennyshaped connection given by

$$
k_{2}=\frac{Q \cos \phi}{2 \pi a^{3 / 2}}, k_{3}=-\frac{Q \sin \phi}{2 \pi a^{3 / 2}}
$$

Figures 2 and 3 show the results of $k_{2}$ and $k_{3}$ in equation (27) in the form of curves. It is seen that $k_{2}$ decreases in magnitude as the angle $\phi$ is increased from $0^{\circ}$ to $90^{\circ}$ at which $k_{2}$ vanishes. On the other hand, since a state of pure shearing prevails at $\phi=0^{\circ}, k_{3}=0$. At other points of the crack border, both $k_{2}$ and $k_{3}$ are present. However, their combined effect leading to brittle fracture is not known as a priori. For this reason, 
the shape into which the elliptical bond would spread under the action of shear can only be determined experimentally by assuming that the function

$$
f\left(k_{2}, k_{3}\right)=f_{c r}
$$

reaches certain critical value at the onset of crack propagation.

When the elliptical bond is sheared along the minor axis with resultant force $R$, the same procedures may be employed to find the stress-intensity factors

$$
\begin{aligned}
& k_{2}=\frac{R}{2 \pi} \sqrt{\frac{a}{b}} \sin \phi\left(a^{2} \sin ^{2} \phi+b^{2} \cos ^{2} \phi\right)^{-3 / 4} \\
& k_{3}=\frac{R}{2 \pi} \sqrt{\frac{b}{a}} \cos \phi\left(a^{2} \sin ^{2} \phi+b^{2} \cos ^{2} \phi\right)-3 / 4
\end{aligned}
$$

Equation (28) is strictly analogous to equation (27) and thus no separate comment is required.

\section{REFERENCES}

1. Green, A. E. and Sneddon, I. N., "The Distribution of Stress in the Neighborhood of a Flat Elliptical Crack in an Elastic Solid", Proc. Cambridge Phi1. Soc., Vol. 46, pp. 159-164, (1950).

2. Kassir, M. K. and Sih, G. C., "Three-Dimensional Stress Distribution Around an Elliptical Crack Under Arbitrary Loadings", J. Appl. Mech. Vol. 33, pp. 601-611, (1966). 
3. Kassir, M. K. and Sih, G. C., "Geometric Discontinuities in Elastostatics", J. Math. Mech. Vo1. 16, pp. 927-948, (1967).

4. Kassir, M. K. and Sih, G. C., "Some Three-Dimensional Inclusion Problems in Elasticity", Int. J. Engng. Sci. (in Press).

5. Westmann, R. A., "Note on Estimating Critical Stress for Irregularly-Shaped-Planar Cracks", Int. J. Fracture Mech. Vol. 2, pp. 561-563, (1966).

6. Irwin, G. R., "Crack-Extension Force for a Part-Through Crack in a Plate", J. Appl. Mech. Vol. 29, pp. 651-654, (1962).

TITLE OF FIGURES

Figure 1 - Stress-Intensity Factor For Opening Mode.

Figure 2 - Stress-Intensity Factor For Shearing Mode.

Figure 3 - Stress-Intensity Factor For Tearing Mode. 


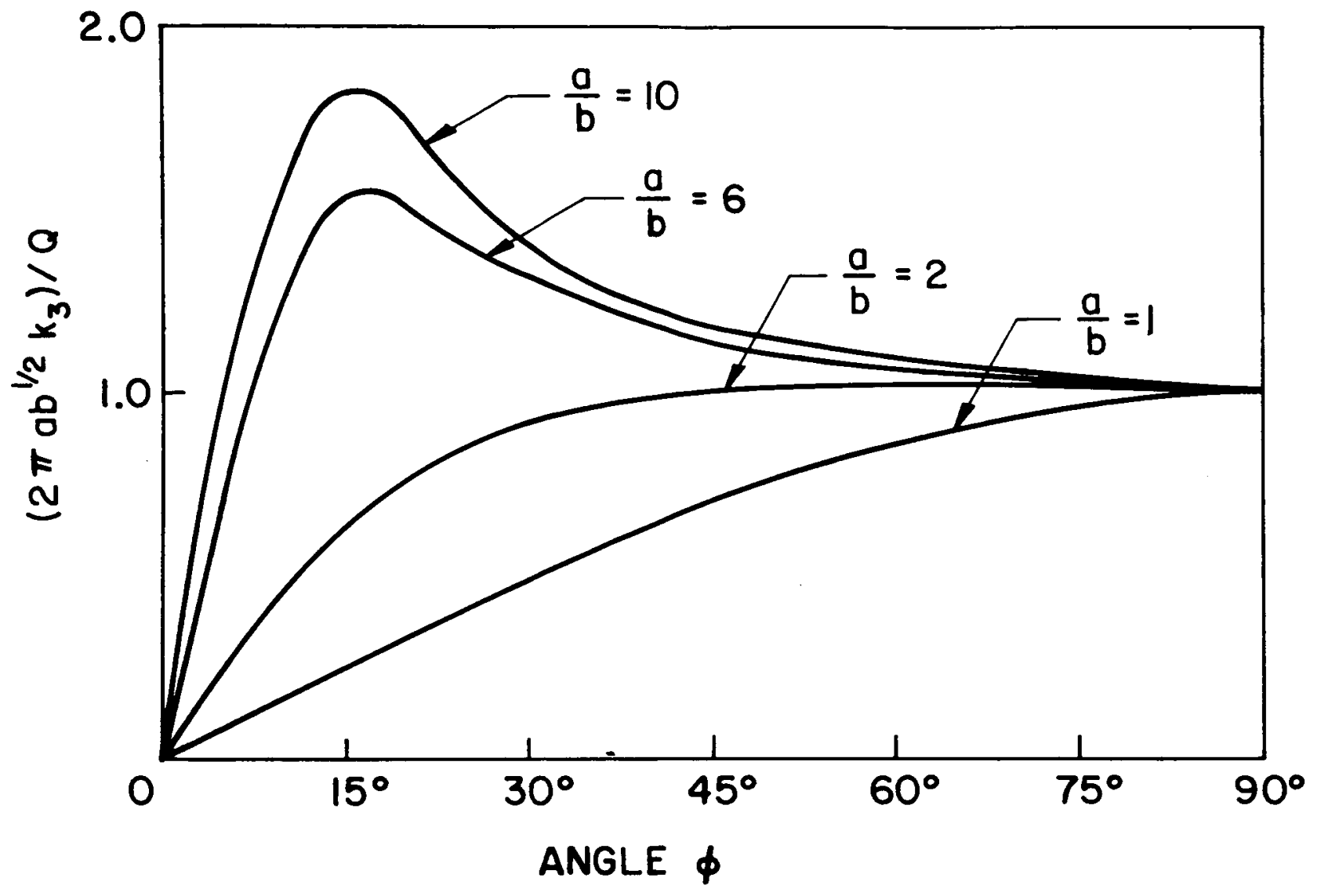




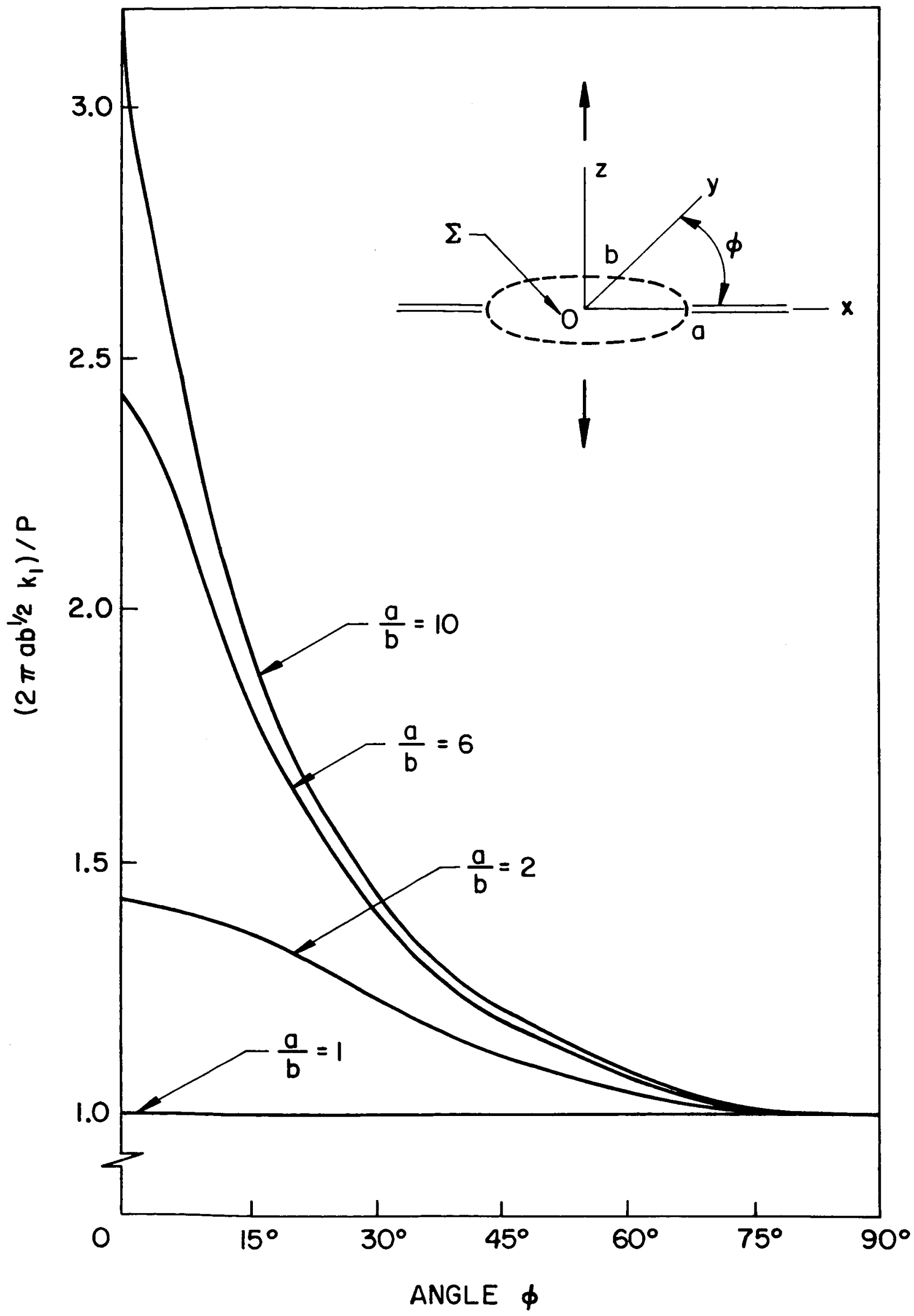




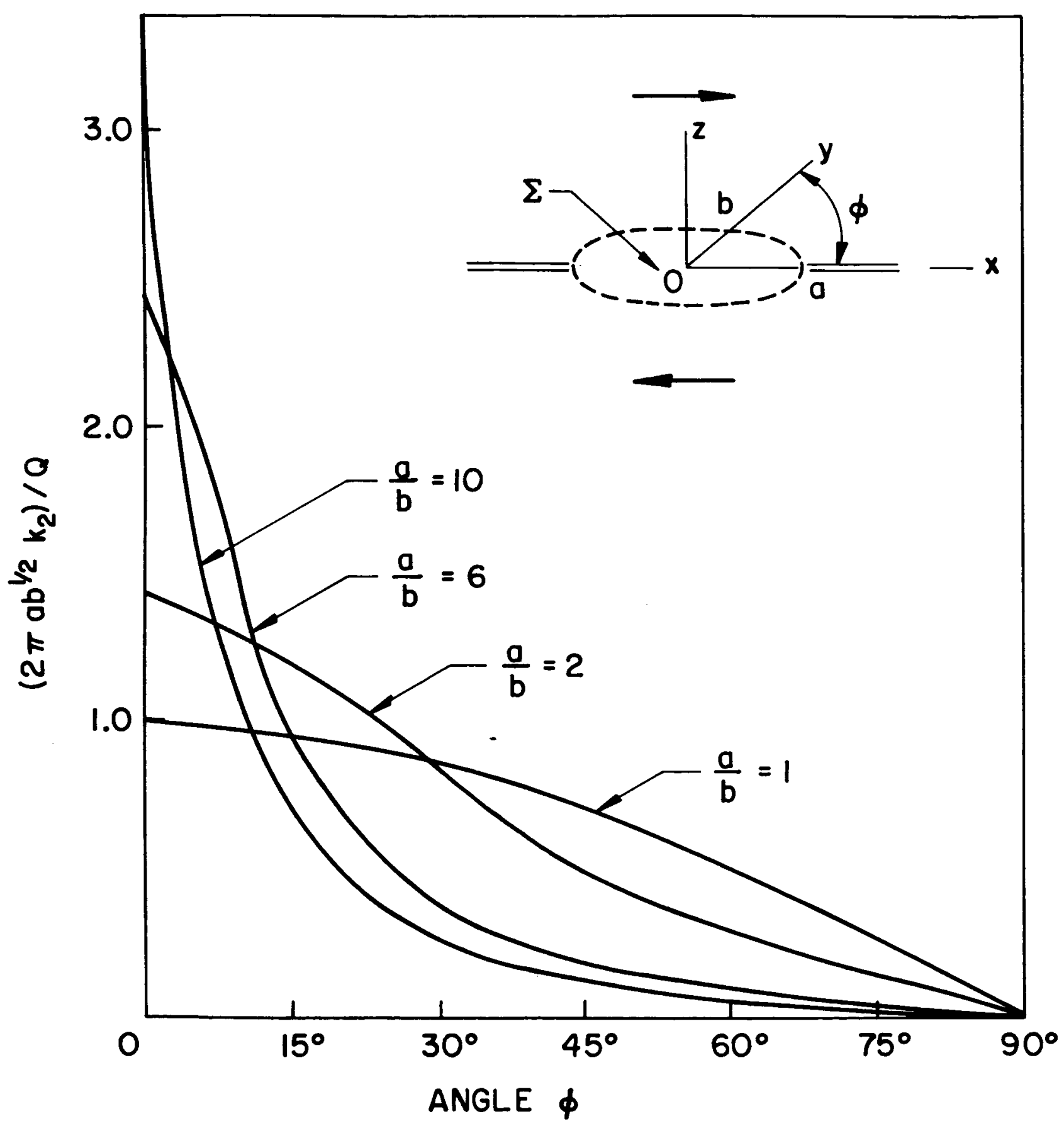

